Maximilian H. Grafmüller

Prozeßmanagement in der Automobilindustrie 
Maximilian H. Grafmüller

\section{Prozeßmanagement in der Automobilindustrie}

Betriebliche Umsetzung am Beispiel der Volkswagen AG

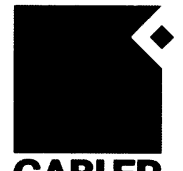

GABLER 
1. Auflage November 2000

Alle Rechte vorbehalten

(C) Springer Fachmedien Wiesbaden 2000

Ursprünglich erschienen bei Betriebswirtschaftlicher Verlag Dr. Th. Gabler $\mathrm{GmbH}_{\text {, }}$ Wiesbaden 2000

Lektorat: Ulrike M. Vetter / Susanne Kramer

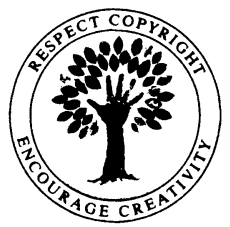

Das Werk einschließlich aller seiner Teile ist urheberrechtlich geschützt. Jede Verwertung außerhalb der engen Grenzen des Urheberrechtsgesetzes ist ohne Zustimmung des Verlages unzulässig und strafbar. Das gilt insbesondere für Vervielfältigungen, Übersetzungen, Mikroverfilmungen und die Einspeicherung und Verarbeitung in elektronischen Systemen.

www.gabler.de

Höchste inhaltliche und technische Qualität unserer Produkte ist unser Ziel. Bei der Produktion und Verbreitung unserer Bücher wollen wir die Umwelt schonen. Dieses Buch ist deshalb auf säurefreiem und chlorfrei gebleichtem Papier gedruckt. Die Einschweißfolie besteht aus Polyäthylen und damit aus organischen Grundstoffen, die weder bei der Herstellung noch bei der Verbrennung Schadstoffe freisetzen.

Die Wiedergabe von Gebrauchsnamen, Handelsnamen, Warenbezeichnungen usw. in diesem Werk berechtigt auch ohne besondere Kennzeichnung nicht zu der Annahme, daß solche Namen im Sinne der Warenzeichen- und Markenschutz-Gesetzgebung als frei zu betrachten wären und daher von jedermann benutzt werden dürften.

Umschlaggestaltung: Nina Faber de.sign, Wiesbaden 


\section{Geleitwort}

Ein „Nachdenken“ über ein Prozeßmanagement in der Unternehmung und möglicherweise gerade in der Automobildistribution, im Duktus einer theoretischen Fundierung, einer Fortentwicklung vorhandener Konzepte, einer empirischen Beleuchtung und einer Fortführung erster betrieblicher Umsetzungsanstrengungen ist dringend notwendig, aber auch höchst kompliziert. Grundlegende Entwicklungen wie zunehmende Sättigung der Märkte, Überkapazitäten, der Wandel von einem Verteiler- zu einem Käufermarkt, die abnehmende Bindungsbereitschaft und die individualisierten Kundenwünsche fordern tiefgreifende Veränderungen gerade in der Distribution, da erhebliche Verbesserungen in den vorgelagerten Bereichen der Wertschöpfungskette - wie Einkauf und Produktion - bereits realisiert worden sind.

Um nachhaltige Erfolge zu erzielen, scheiden kurzfristig orientierte Kostensenkungs-, Ablaufoptimierungs- und Restrukturierungsprojekte wegen ihres „Strohfeuercharakters“ aus. Eine grundlegende Transformation mit einer dauerhaften Erfolgswirkung könnte vielmehr in der Idee liegen, von der viele Schnittstellen produzierenden funktionalen Gestaltung des Unternehmungsgeschehens abzugehen und sich generell an der Wertschöpfungskette, letztlich an kundenzentrierten Prozessen zu orientieren. Eine entsprechende Identifikation, Gestaltung und Implementation und schließlich ein Prozeßmanagement in der Distribution scheint dementsprechend zielführend, wenn an die Erfahrungen in anderen Branchen und Bereichen gedacht wird.

Mit dem bei der Volkswagen AG (VW AG) implementierten und maßgeblich von López de Arriortúa geprägten System einer „Schlanken Produktion“ entlang der Wertschöpfungskette steht zunächst der Zulieferbereich im Fokus. Seit 1993 wurde die Kernidee des kontinuierlichen Verbesserungsprozesses (KVP) - von der Produktionszentrierung kommend - als Gesamtkonzept zur Wertschöpfungskettenoptimierung weiterentwickelt $\left(\mathrm{KVP}^{\mathrm{n}}\right)$. Eine flächendeckende Anwendung auch im Distributionsbereich steht allerdings noch weitgehend aus. Genau hier setzt Herr Grafmüller an, wobei die Herausforderung darin liegt, auf der Basis vorliegender Verfahren und Vorgehensweisen aus dem Produktionsbereich eine entsprechende Transformationsleistung $\mathrm{zu}$ erbringen, um Verkaufs-, Verwaltungs- und weitere Dienstleistungsprozesse unter der Zielrichtung von Qualität, Zeit und Kosten zu optimieren.

Mit dem differenziert ausformulierten und hinsichtlich seiner Herleitung gut begründeten Rahmenkonzept und der bemerkenswerten Ergebnisse der empirischen Beleuchtung bei der VW AG sowie der Skizzierung weiterer Entwicklungspotentiale einer prozeßzentrierten Transformation der Distribution, die dann auch auf weitere Wertschöpfungskettenbereiche ausstrahlen wird, liegt insgesamt ein sehr gut gestalteter Basisstein für die gegenwärtig bestehende Lücke im Argumentationsfeld einer Prozeßorientierung vor.

Ich wünsche diesen Überlegungen eine weite Verbreitung. 


\section{Vorwort}

Die prozeßorientierte Optimierung der Vertriebsorganisation stellt für die Automobilindustrie eine entscheidende Herausforderung dar. Einen wertvollen Beitrag zur Bewältigung dieser Herausforderung liefert das vorliegende Werk von Maximilian Grafmüller.

Es wird darin erstmals das weltweit erfolgreich angewandte $\mathrm{KVP}^{8}$-Konzept zur Verbesserung von Prozeßabläufen in der Automobildistribution und zur Transformation von funktionalen in prozeßorientierte Organisationsstrukturen wissenschaftlich durchleuchtet.

Basierend auf mehrdimensionalen Analysen von über $2500 \mathrm{KVP}^{8}$-Workshops in den Vertriebssystemen des Volkswagen-Konzerns werden Erfolgsstrategien und Entwicklungspotentiale identifiziert, die auch für den Praktiker von Bedeutung sind.

Dr. Ing. Jóse Ignacio López de Arriortúa 


\section{INHALTSVERZEICHNIS}

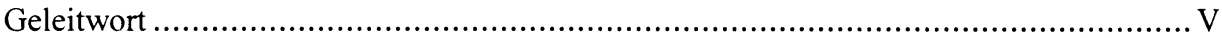

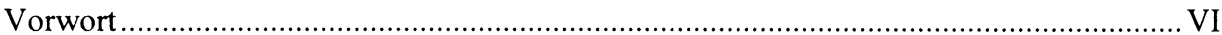

1. Einführung: Prozeßmanagement in der Automobildistribution als betriebswirtschaftlicher Forschungsgegenstand ................................ 1

1.1 Die Notwendigkeit eines tiefgreifenden Wandels in der Automobildistribution als Ansatzpunkt für neue Entwicklungsmöglichkeiten ................ 1

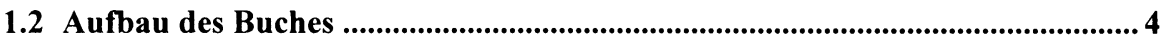

2. Grundlagen und Ansatzpunkte für die prozeßorientierte Organisationsgestaltung....................................................................................... 6

2.1 Zur Weiterentwicklung bestehender Prozeßmanagement-Ansätze auf der Basis eines systemtheoretischen Grundgerüsts ................................................6 6

2.1.1 Potentiale für eine Evolution prozeßorientierter Organisationskonzepte ...... 6

2.1.2 Begriffliche Erläuterungen zum Organisationsverständnis......................... 7

2.2 Systemtheoretische Ausgangspunkte für Selbstorganisation und

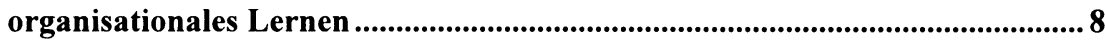

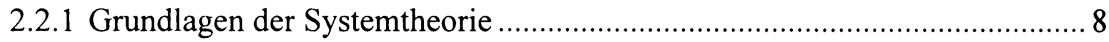

2.2.2 Selbstorganisation als evolutorischer Prozeß ........................................ 10

2.2.3 Selbstorganisation und organisationaler Wandel ......................................... 12

2.2.4 Organisationales Lernen: Systematische Wissensgenerierung im Einklang mit der Entwicklung von Gebrauchs- und Handlungstheorien..... 14

2.2.5 Kreativitätstechniken zur Förderung von Selbstorganisation und organisationalem Lernen .................................................................... 19

2.2.5.1 Das Phänomen Kreativität........................................................... 19

2.2.5.2 Kreativitätstechniken und ihre Wirkmechanismen ....................... 20

2.3 Grundverständnis der Prozeßorganisation ......................................................22

2.3.1 Bestimmung des Prozeßbegriffs im organisationalen Bezug ..................... 22

2.3.2 Grundformen der Prozeßanalyse: Prozeßausgrenzung, -zerlegung und

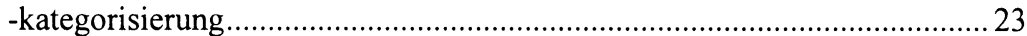

2.3.3 Begriffsbildung und Anwendungsbereich der Prozeßorganisation.............. 27

2.4 Merkmale der Prozeßorganisation im Verhältnis zur „klassischen“ betriebswirtschaftlichen Organisationslehre .28

2.4.1 Aufbau- und Ablauforganisation als spezifische Inhaltskategorien der deutschen Organisationslehre .................................................................. 28

2.4.2 Kundenorientierung und wertschöpfungskettenbezogene Betrachtungsweise als neue Beiträge zur Prozeßorganisation 30 
2.5 Kundenorientierung in der Prozeßorganisation.

2.5.1 Das Prinzip des internen und externen Kunden als Ausdruck eines neuen Kundenverständnisses.

2.5.2 Kundenzufriedenheit: Die Zielgröße der kundenorientierten

Prozeßorganisation

2.5.3 Kundenzufriedenheitsmessung als Ausgangspunkt für kontinuierliche Verbesserungen in der Prozeßorganisation ...

2.5.4 Die Erfolgspotentiale der Kundenzufriedenheit im Rahmen eines auf den gesamten Besitzlebenszyklus des Kunden ausgerichteten Betreuungsansatzes .

2.6 Prozeßorganisation und ganzheitliche Wertschöpfungskettenperspektive

2.6.1 Das Wertkettenkonzept von Porter als Beginn einer Fokussierung auf kundenbezogene Wertschöpfungsprozesse.

2.6.2 Die Wertschöpfungskette als Gerüst für die Ausbildung strategischer Netzwerke

2.6.3 Strategische Netzwerke als „hyperoffene Systeme“

2.6.4 Strategische Netzwerke und Selbstorganisation

2.6.5 Zur Generierung von Erfolgspotentialen der Wertschöpfungskette durch netzwerkorientiertes Prozeßmanagement.

2.7 Zwischenbilanz: Aspekte prozeßorientierter Organisationsgestaltung auf der Grundlage eines systemtheoretischen Bezugsrahmens.

3. Das Management der Prozeßorganisation

3.1 Grundannahmen zur Managementkonzeption.

3.2 Die Zielgrößen des Prozeßmanagements und deren Bezug zum

Ergebnisparameter Kundenzufriedenheit.

3.2.1 Qualität: „First-Time-Quality“ aus Kundensicht erzeugen......................... 50

3.2.2 Kosten: Die Prozeßkostenrechnung als Grundlage für

Kostentransparenz und kundenorientierte Prozeßflexibilität.....

3.2.3 Zeit: Antizipation und konsequentes Zeitmanagement als Chance in einem Umfeld beschleunigten Wandels.....

3.2.4 Die Zielgrößen und ihre Ausrichtung am Ergebnisparameter

Kundenzufriedenheit .....

3.3 Prozeßsteuerung auf der Basis eines ganzheitlichen

Prozeßkennzahlensystems.

3.3.1 Verhaltenssteuerndes Controlling als Grundlage für ein Informationskonzept zur Prozeßsteuerung.

3.3.2 Aspekte für den Aufbau eines ganzheitlichen Prozeßkennzahlensystems.

3.4 Prozeßgestaltung im Rahmen des Prozeßmanagements

3.4.1 Das kundenorientierte Unternehmungsprozeßmodell als Ansatz zur Prozeßidentifikation.

3.4.2 Prozeßstrukturdarstellung: Motivation, Anforderungen und Ziele

3.4.3 Prozeßdekomposition: Entwicklung einer hierarchischen

Prozeßstruktur vom Kern- zum Elementarprozeß. 
3.5 Aufbauorganisatorische Integration des Prozeßmanagements....................... 72

3.5.1 Ansatzpunkte für eine organisatorische Verankerung des

Prozeßmanagements

3.5.2 Matrix- und Projektorganisation als Basis für die Strukturmodelle der

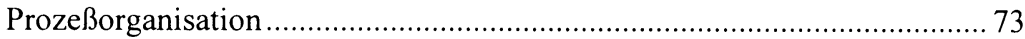

3.5.2.1 Die Matrixorganisation als Träger der Prozeßdimension................. 73

3.5.2.2 Grundformen der Projektorganisation als Strukturmuster für

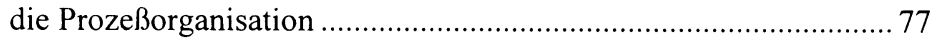

3.5.3 Ein- und mehrdimensionale Formen der Prozeßorganisation ..................... 79

3.6 Gruppenkonzepte in der prozeßorientierten Arbeitsorganisation ..................8 83

3.6.1 Aspekte zur Leistungseffizienz der Gruppenarbeit ................................... 83

3.6.2 Grundformen der Arbeitsgruppe in der Prozeßorganisation ......................... 85

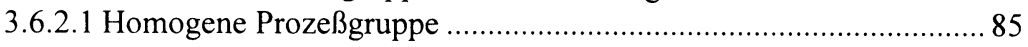

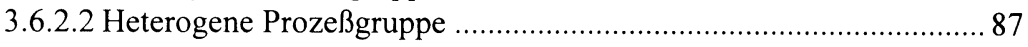

3.7 Ein gewandeltes Führungsverständnis als Leitbild der prozeßorientierten Arbeitsorganisation ........................................................88

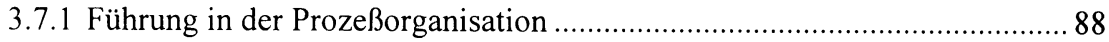

3.7.2 Der Prozeßverantwortliche und seine Führungsaufgabe ........................... 90

3.8 Zwischenbilanz: Das Prozeßmanagementkonzept als Grundlage einer objektspezifischen, ganzheitlichen Veränderungsstrategie

\section{Distributionssysteme der Automobilindustrie als Objekt von} Prozeßorganisation und -management...................................................... 92

4.1 Grundlagen der Automobildistribution .........................................................92

4.1.1 Inhaltliche und begriffliche Abgrenzung der Distribution im Rahmen des Absatzes von Automobilen ............................................................... 92

4.1.2 Aufbau des Distributionssystems ............................................................ 95

4.1.3 Aktionsbereiche der betrieblichen Distributionspolitik zur Umsetzung der Distributionsstrategie

4.2 Strukturanalyse der Distributionssysteme im deutschen Automobilmarkt

4.2.1 Der institutionenorientierte Ansatz als Ausgangspunkt der Distributionsanalyse

4.2.2 Die Aufbauorganisation der herstellereigenen Distributionsorgane ............ 98

4.2.3 Der selektive Vertrieb als Distributionsform im deutschen Automobilmarkt

4.2.4 Die Grundformen der Distributionslogistik deutscher Hersteller ...

4.2.5 Der Vertragshändler und seine Aufgaben im Distributionssystem 104

4.3 Problemfelder der Automobildistribution: Von Symptomen zu

Ursachen und Erklärungsansätzen.

4.3.1 Rentabilitätsprobleme in den Distributionssystemen durch eine sprunghafte Zunahme der Wettbewerbsdynamik

4.3.2 Die Wertschöpfungskette als gespaltenes System: Vom „Pull-System“ im Einkauf zum „Push-System“ in der Distribution.

4.3.3 Bidirektionales Informationsgefälle innerhalb der Wertschöpfungskette 
4.4 Gestaltungspotentiale des Prozeßmanagements in der

Automobildistribution

4.4.1 Produktzentrierte Komplexitätsreduktion als Basis der prozessualen

Effizienzsteigerung.

4.4.2 Neuausrichtung des logistischen Distributionsystems bei Fokussierung auf den Prozeß „Kunde bis Kunde“

4.4.2.1 „Lagerlose“ Vertragshändler als erster Schritt zur

Transformation vom „Stock-Push“- zum „Stock-Pull“-System .... 112

4.4.2.2 Die zweite Stufe der Prozeßoptimierung mit dem Ziel der

Implementierung eines reinen Auftragsfertigungssystems............ 114

4.4.2.3 Die Auftragsdurchlaufzeitoptimierung bei der VW AG ............... 116

4.4.3 Komplexitätsreduktion im akquisitorischen Distributionssystem ............. 116

4.4.4 Das Autohaus und die Nutzenpotentiale des Prozeßmanagements........... 119

4.4.4.1 Ansatzpunkte für ein Prozeßmanagement im Autohaus ............... 119

4.4.4.2 Die Cluster-Organisation im prozeßorientierten Autohaus ............ 120

4.4.4.3 Kundenbindung durch Clustering ............................................ 122

4.4.4.4 Kundenbindung über neue Arbeitsmodelle in der

Werkstattorganisation

4.4.4.5 Prozeßleistungstransparenz im Autohaus als Voraussetzung für eine differenzierte Kundenansprache

4.5 Zwischenbilanz: Die Analyse der Automobildistribution in bezug auf Anwendungspotentiale des Prozeßmanagements.

5. Die Entwicklung des $\mathrm{KVP}^{8}$-Konzeptes zur betrieblichen Umsetzung des Prozeßmanagements im Distributionssystem der VW AG

5.1 Grundlagen des $\mathrm{KVP}^{8}$-Konzeptes

5.1.1 Einordnung des $\mathrm{KVP}^{8}$-Konzeptes in das $\mathrm{KVP}^{\mathrm{n}}$-Gesamtkonzept zur

Wertschöpfungskettenentwicklung

5.1.2 Ansatzpunkte und Forschungsmethodik bei Entwicklung des $\mathrm{KVP}^{8}$ -

Konzeptes

5.1.3 Das $\mathrm{KVP}^{8}$-Konzept als integrativer Ansatz der Managementkonzepte des prozeßorientierten Wandels

5.2 Gestaltung und Steuerung des Prozeßmanagements im Vertragshandel durch das $\mathrm{KVP}^{\mathbf{8}}$-Konzept.

5.2.1 Analyse der Kernprozesse des Vertragshändlers anhand eines kundenorientierten Unternehmungsprozeßmodells

5.2.2 Das KVP ${ }^{8}$-Prozeßkennzahlensystem: Verhaltenssteuerndes

Controlling unter dem Aspekt der Elimination von „Verschwendung“

in Dienstleistungsprozessen

5.3 Grundlagen des $\mathrm{KVP}^{8}$-Workshops als Gestaltungsinstrument für die Implementierung von Selbstorganisation und prozeßorientiertem

Wandel

5.3.1 Die Philosophie des KVP ${ }^{8}$-Workshops

5.3.2 Kreativitätstechniken im $\mathrm{KVP}^{8}$-Workshop: „Destruktiv-konstruktives“

Brainstorming als Instrument der Alternativensuche 
5.4 Der KVP ${ }^{8}$-Basisworkshop: Ein multiplikativer, bottom-up orientierter Ansatz zur evolutionären Prozeßverbesserung

5.4.1 Die Vorbereitungsphase zur Einbindung von Mitarbeitern und Management in den kontinuierlichen Verbesserungsprozeß

5.4.1.1 Der KVP ${ }^{8}$-Führungskräfte-Workshop ........................................... 144

5.4.1.2 Das Vorgespräch zum KVP ${ }^{8}$-Workshop ...................................... 146

5.4.2 Der KVP ${ }^{8}$-Basisworkshop in der Phasenanalyse .................................... 147

5.4.3 Workshop-Nachbetreuung und Übergang in ein kontinuierliches Prozeßverbesserungsprogramm

5.5 Revolutionäre, innovative Prozeßverbesserung mit dem Instrument des KVP $^{\mathbf{8}}$-Reorganisationsworkshops

5.5.1 Der konzeptionelle Ansatz des $\mathrm{KVP}^{8}$-Reorganisationsworkshops ............ 154

5.5.2 Der $\mathrm{KVP}^{8}$-Reorganisationsworkshop am Beispiel „Systematische

Marktbearbeitung im Fahrzeugverkauf"

5.5.2.1 Nutzenpotentiale eines neugestalteten Prozeßablaufs im Fahrzeugverkauf.

5.5.2.2 Phasenablauf und Module des KVP ${ }^{8}$-Reorganisationsworkshops.

5.6 Zwischenbilanz: Philosophie und Bausteine des KVP ${ }^{8}$-Konzeptes

6. Gestaltungshinweise für die Weiterentwickung des $\mathrm{KVP}^{8}$ Konzeptes auf der Basis empirischer und mehrebenenanalytischer Untersuchungen

6.1 Empirische, mehrdimensionale und multinationale Analyse des $\mathrm{KVP}^{8}$-Konzeptes

6.1.1 Vorgehensweise und Methodik bei der empirischen $\mathrm{KVP}^{8}$-Analyse ......... 167

6.1.2 Prozeßmeßgrößenanalyse der in das $\mathrm{KVP}^{8}$-Konzept integrierten Vertragshändler

6.1.3 Die Bewertung des KVP ${ }^{8}$-Konzeptes durch die Vertragshändler als interne Kunden.

6.1.4 Das KVP ${ }^{8}$-Konzept und die Zufriedenheit des Endkunden:

Multinationale Analyse bestehender Kundenzufriedenheitsindices, bezogen auf die Durchführung von $\mathrm{KVP}^{8}$-Workshops ...

6.1.4.1 Vorgehensweise und Ziele der Untersuchung....

6.1.4.2 Die Kundenzufriedenheitsentwicklung durch $\mathrm{KVP}^{8} \mathrm{im}$ spanischen Automobilmarkt.

6.1.4.3 Die Auswirkungen der KVP ${ }^{8}$-Aktivitäten auf die Kundenzufriedenheit im deutschen Automobilmarkt mit einer gesonderten Betrachtung für den $\mathrm{KVP}^{8}$-Reorganisationsworkshop.

6.1.4.4 Die Beeinflussung der Kundenzufriedenheit durch KVP ${ }_{-}$ Workshops im französischen Automobilmarkt.

6.1.4.5 $\mathrm{KVP}^{8}$ und erste Ergebnisse zur Kundenzufriedenheitsentwicklung im amerikanischen Automobilmarkt 
6.2 Das KVP ${ }^{8}$-Konzept in der mehrebenenanalytischen Betrachtung................. 192

6.2.1 Steinles Mehr-Ebenen-Analyse als Vorgehenspfad ................................ 192

6.2.2 Betrachtungsebene Umwelt: Das $\mathrm{KVP}^{8}$-Konzept und die UmweltHarmonisation in allen Stufen der Wertschöpfungskette.

6.2.3 Betrachtungsebene Unternehmung: Die Wirkungszusammenhänge des $\mathrm{KVP}^{8}$-Konzeptes für eine schrittweise Entwicklung von der funktionalen Organisation zur lernenden Prozeßorganisation .....

6.2.4 Betrachtungsebene Gruppe: Der KVP ${ }^{8}$-Workshop als Baustein für die organisationale Wissensbildung

6.2.5. Betrachtungsebene Individuum: Der Mitarbeiter als Protagonist im

Lernprozeß von der Fremd- zur Selbstorganisation .

6.3 Entwicklungspotentiale des $\mathrm{KVP}^{8}$-Konzeptes auf der Basis des herausgearbeiteten empirisch-theoretischen Grundgerüsts .

6.3.1 Ansatzpunkte für die zukünftige Konzeptentwicklung.

6.3.2 Verbesserungspotentiale des $\mathrm{KVP}^{8}$-Konzeptes zur Anwendung im

Vertragshandel der Automobilindustrie

6.3.2.1 Entwicklung von $\mathrm{KVP}^{8}$-Reorganisationsworkshops für eine ganzheitliche Transformation der Kundendienstprozesse des Autohauses

6.3.2.2 $\mathrm{KVP}^{8}$-Reorganisationsworkshops zur Implementierung der Cluster-Organisation im Autohaus

6.3.3 Das $\mathrm{KVP}^{8}$-Konzept als Prozeßentwicklungsinstrument für alle Prozesse der akquisitorischen und physischen Automobildistribution...... 203

6.3.3.1 Prozessuale Leistungssteigerungspotentiale der Automobildistribution als Ausgangspunkt für die konzeptionelle Weiterentwicklung ....

6.3.3.2 Ausrichtung des Projektmanagements an der Komplexität der Zielorganisation.

6.3.3.3 Konzeptionelle Integration von Mitarbeiterzufriedenheitsanalysen, Learning Maps und Sensitivitätsmodellen

6.3.3.4 Einbindung eines EDV-Systems zur Prozeßmodellierung......

6.3.3.5 $\mathrm{KVP}^{8}$-Reorganisationsworkshops als ganzheitliches Gestaltungs- und Implementierungsinstrument zur Einführung von Workflow-Management-Systemen..

6.3.3.6 Integration der $\mathrm{KVP}^{8}$-Aktivitäten in Entwicklung und Implementierung von SAP R $/ 3^{\circledR}$ in der Automobilindustrie.

6.3.3.7 Entwicklung von $\mathrm{KVP}^{8}$-Prozeßkostenworkshops auf der Grundlage einer integrierten Prozeßmeßgrößenerfassung.

\subsection{Zwischenbilanz: Entwicklungspotentiale für das $\mathrm{KVP}^{\mathbf{8}}$-Konzept auf der Basis empirisch-theoretischer Analyse.}

7. Die Ergebnisse im Überblick

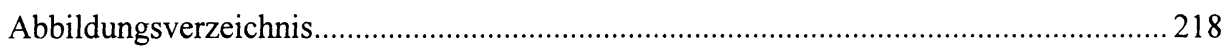

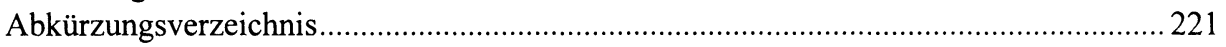

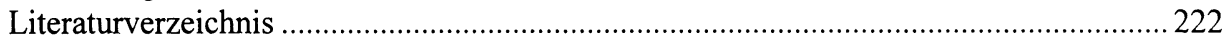

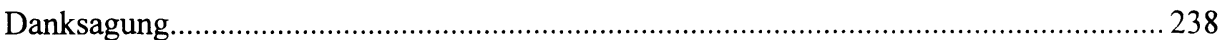

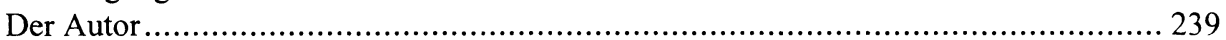

